\title{
An Undulating Tongue: Post-Irradiation Myokymia and Neuromyotonia of the Tongue Following Radiation Therapy for Tonsillar Cancer
}

\section{Abstract}

Delayed complications of radiation therapy affecting the cranial nerves are quite rare. We report a case of tongue myokymia and neuromyotonia following radiation therapy for tonsillar cancer.

Case report: 62-year-old male presented with progressive dysarthria, dysphagia and tongue paralysis and myokymia 10 years after irradiation for tonsillar cancer. Electro diagnostic studies showed myokymic and neuromyotonic discharges, while brain and neck MRI were unremarkable, ruling out tumor recurrence.

Conclusion: Delayed neuropathy of CN XII with tongue myokymia is a rarely reported complication of radiation therapy. Clinicians should be aware of this condition to prevent misdiagnosis.

Keywords: Cancer; Myokymia; Neuromyotonia; Radiation; Tongue

Received: April 09, 2018; Accepted: May 16, 2018; Published: May 23, 2018

\section{Introduction}

Myokymia is a clinical phenomenon presenting as continuous involuntary undulation or rippling of the muscles with electrophysiological evidence of myokymic discharges. Radiation therapy can affect both central and peripheral nervous system, with the latter being much more common, often presenting as brachial or lumbosacral plexopathy with myokymia. Myokymia secondary to post-irradiation cranial neuropathies is much less common, with only few published reports. We report a case of delayed post-irradiation myokymia and neuromyotonia, confirmed electrodiagnostically, in patient with history of tonsillar cancer.

\section{Case Report}

A 62-year-old male presented with a two-year-history of progressive dysarthria, dysphagia, and limited motion of the tongue in all directions. He received radiation therapy for tonsillar cancer 10 years prior. Neurological exam showed dysarthria and lingual atrophy, especially on the right, with continuous irregular undulating movements in the right side of his tongue. Fasciculations were also noted in the left upper trapezius muscle.
Fabian Rossi ${ }^{1}$, Welwin Liü2*, Nina Tsakadze ${ }^{1}$, Michael Hoffmann', Ramon Rodriguez-Cruz ${ }^{1}$, Elisa Rossi", Elizabeth Gonzalez ${ }^{1}$, Stephen Castaneda ${ }^{1}$, Aunali Khaku ${ }^{1}$, Maria Franco ${ }^{3}$ and Alvaro Estevez ${ }^{3}$

\section{Department of Neurology, Orlando VA Medical Center, 13800 Veterans Way, Orlando, FL 32827, USA \\ 2 Department of Neurology, Spark M. Matsunaga VA Medical Center, 459 Patterson Road, ACC Module 8, Honolulu, HI 96819, USA \\ 3 Department of Biochemistry and Biophysics, Oregon State University, ALS 2103, Corvallis, OR 97331, USA}

\section{*Corresponding author: Welwin Liu}

巨Welwin.Liu@va.gov

Spark M. Matsunaga VA Medical Center, Department of Neurology, 459 Patterson Road, Honolulu, HI 96819, USA.

Tel: +9999744771

Citation: Rossi F, Liu W, Tsakadze N, Hoffmann M, Rodriguez-Cruz R, et al. (2018) An Undulating Tongue: Post-Irradiation Myokymia and Neuromyotonia of the Tongue Following Radiation Therapy for Tonsillar Cancer. Med Clin Rev Vol.4 No.2:6
The rest of the neurological examination was unremarkable. Needle electromyography (EMG) of the tongue revealed myokymic and neuromyotonic discharges, but no fibrillations, positive sharp waves, or fasciculations. Needle EMG of the right upper and lower limb muscles was normal. Neck and head MRI were also unremarkable, ruling out recurrence of the cancer. 


\section{Discussion and Conclusion}

Myokymia is a spontaneous repetitive discharge of one or a few motor units at regular intervals. It presents clinically as a continuous and irregular wave-like rippling of the skin or mucosa overlying the affected muscles. Neuromyotonia is a spontaneous burst of continuous electrical discharge firing at a frequency up to $300 \mathrm{~Hz}$, lasting up to seconds, rhythmical, and with an abrupt onset and offset. Post-irradiation myokymia and neuromyotonia of the tongue is extremely rare and seldom reported in literature [1-3]. As reported in this case, patient presented with dysarthria, dysphagia, and tongue atrophy with continuous undulating movements of the tongue (Figure 1) (Video 1).

Some patients display facial myokymia [2]. Myokymia and neuromyotonia may present several months or years after irradiation with interval between 10 months [4] to 14 years [5]. Earlier onset of symptoms may be related to more direct radiation exposure or to higher dose of radiation applied. In 4 of the 6 reported cases, myokymia and neuromyotonia followed the treatment for nasopharyngeal carcinoma [1-5], in one other case - radiation therapy for cervical melanoma [6] and in

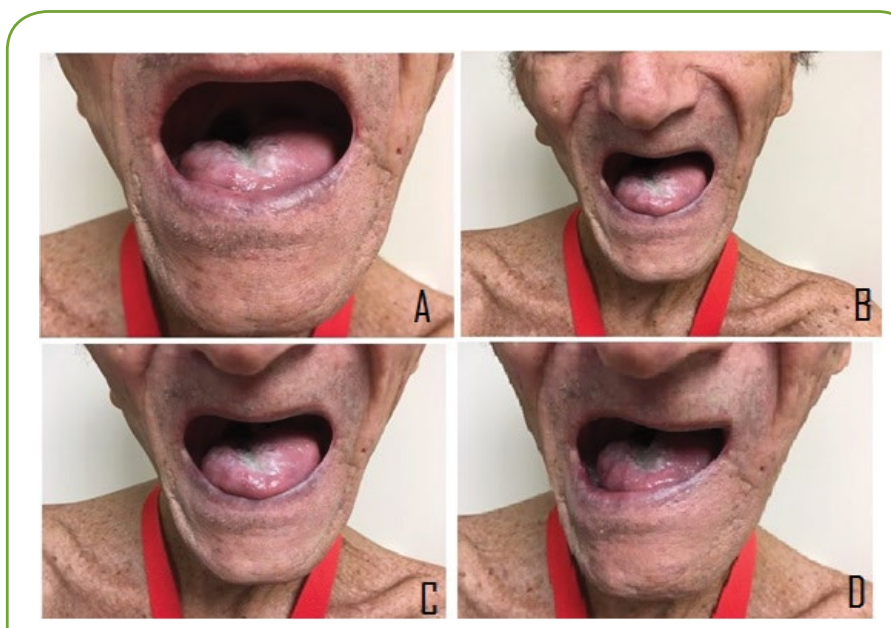

Figure 1 Tongue atrophy (A) Most prominent on the right (B) Patient attempting to protrude his tongue forward (C) To the right and (D) To the left.

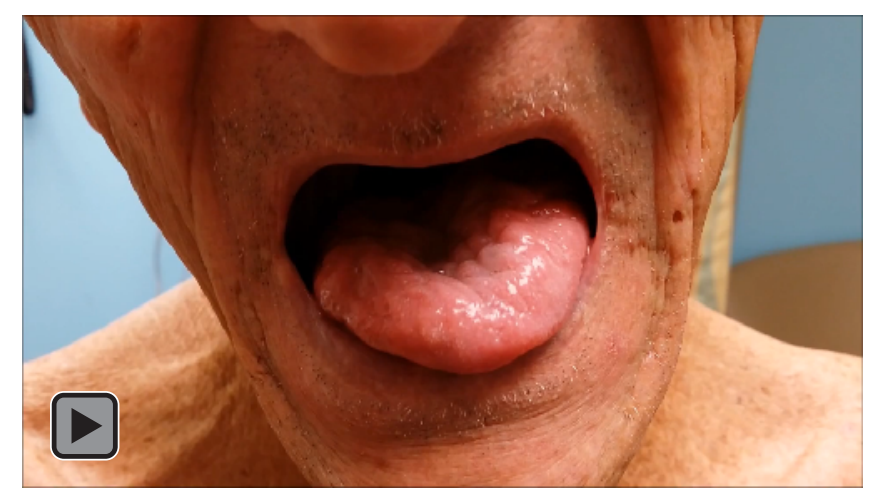

Video 1 Tongue atrophy with irregular rippling and undulating movements (myokymia), more prominent on the right. another - irradiation of the middle ear chemodectoma [7]. In all cases, including current report, needle EMG showed myokymia and neuromyotonic discharges, but no fibrillations or positive sharp waves (Video 2). Fasciculations were reported in one case [8]. Brain MRI with contrast did not show any evidence of tumor recurrence, suggesting irradiation as the most probable etiology. Radiation therapy may injure the hypoglossal nerve and the myokymia and neuromyotonia may result from motor neuron hyperexcitability with ectopic discharges, which reflects the nonspecific neuronal response to injury. Both myokymia and neuromyotonia are thought to originate along segments of the motor axon and are presumed to derive from spontaneous depolarization or ephaptic transmission along segments of demyelinated nerve, secondary to altered membrane excitability [9]. Damage of the peripheral nerve voltage gated $\mathrm{K}+$ channels was also suggested [8]. The post-irradiation hypoglossal nerve damage is probably a process similar to that described in post-irradiation brachial plexopathy [1]. Delayed radiation induced nerve damage is thought to be due to either direct damage to the nerve cells, or alternatively -injury to the vascular endothelium with resultant ischemia, fibrosis and secondary compromise of the neural tissue [10]. Myokymic discharges after radiation therapy indicate radiation-induced neuropathy rather than tumor recurrence or metastatic spread, which makes electrodiagnostic studies valuable in differential diagnosis [3]. Other conditions to consider in differential diagnosis for bulbar myokymia are listed in Table 1. Myokymia sometimes responds to anticonvulsants such as Carbamazepine [4]. To our knowledge, this is the first case of post-irradiation tongue myokymia and neuromyotonia after treatment of tonsillar cancer.

Table 1: Etiology of Bulbar Myokymia.

\begin{tabular}{|c|}
\hline Etiology of Bulbar Myokymia \\
Multiple sclerosis \\
Pontine glioma \\
\hline Guillian-barre syndrome \\
Cardiopulmonary arrest \\
Metastatic tumor \\
\hline Extra-axial posterior fossa lesions \\
\hline Basilar invagination \\
\hline
\end{tabular}

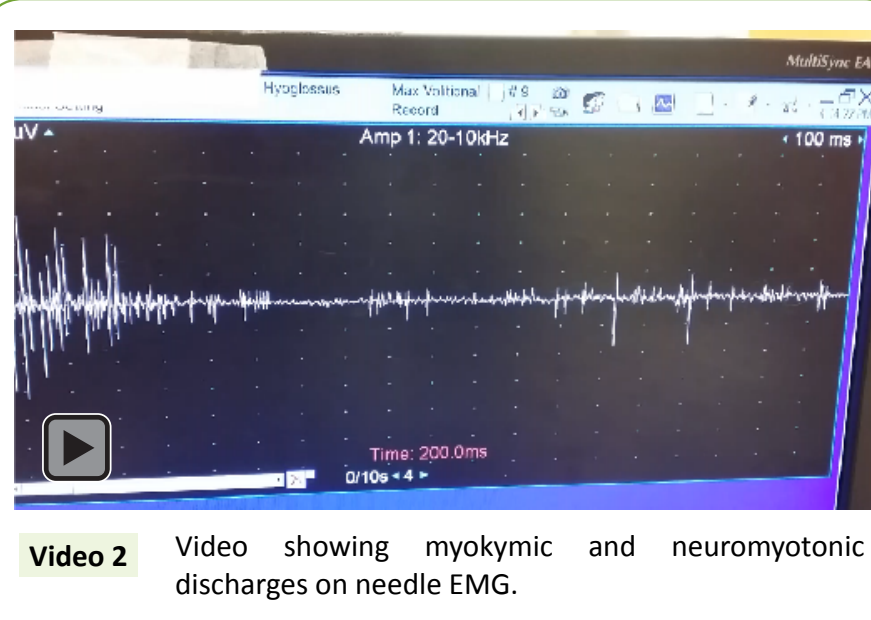

This article is available in http://medical-clinical-reviews.imedpub.com/ 


\section{References}

1 Wang V, Liao KK, Ju TH (1993) Myokymia and neuromyotonia of the tongue: A case report of complication of irradiation. Chung Hua I Hsueh Tsa Chih (Taipei) 52: 413-415.

2 Liu LH, Chen CW, Chang MH (2007) Post-irradiation myokymia and neuromyotonia in unilateral tongue and mentalis muscles: Report of a case. Acta Neurol Taiwan 16: 33-36.

3 Tifticioglu BI, Bulbul I, Ozcelik MM (2016) Tongue myokymia presenting twelve years after radiation therapy. Clin Neurophys Practice 1: 41-42.

4 Rison RA, Beydoun S (2009) Teaching Video Neurolmages: Tongue Myokymia following head and neck radiotherapy for nasopharyngeal carcinoma. Neurology 72: 65.
5 Shapiro B, Rordorf G, Schwamm L, Preston DC (1996) Delayed radiation induced bulbar palsy. Neurology 46: 1604-1606.

6 Richardson R, Weiss M (2009) Unilateral myokymia of the tongue after radiation therapy for cervical nodal melanoma. J Clin Neuromusc Dis 10: 122-125.

7 Poncelet AN, Auger RG, Silber MH (1996) Myokymic discharges of the tongue after radiation to the head and neck. Neurology 46: 259260.

8 Gutmann L, Gutmann L (2004) Myokymia and neuromyotonia 2004. J Neurol 251: 138-142.

9 Gutmann L (1991) AAEM minimonograph 37: Facial and limb myokymia. Muscle Nerve 14: 1043-1049.

10 Berger PS, Bataini JP (1977) Radiation-induced cranial nerve palsy. Cancer 40: 152-155. 